\title{
Ferroelectric Gating of Narrow Band-Gap Nanocrystal Arrays with Enhanced Light Matter Coupling
}

Charlie Gréboval ${ }^{1}$, Audrey Chu', Debora Vale Magalhaes ${ }^{2,3}$, Julien Ramade ${ }^{2,3}$, Junling Qu', Prachi Rastogi ${ }^{1}$, Adrien Khalilii', Sang-Soo Chee ${ }^{1}$, Hervé Aubin ${ }^{4}$, Grégory Vincent ${ }^{5}$, Sara Bals ${ }^{2,3}$, Christophe Delerue ${ }^{6}$, Emmanuel Lhuillier ${ }^{*}$

${ }^{1}$ Sorbonne Université, CNRS, Institut des NanoSciences de Paris, INSP, 75005 Paris, France.

2 Electron Microscopy for Materials Science, University of Antwerp, Groenenborgerlaan 171, B2020 Antwerp, Belgium.

${ }^{3}$ NANOlab Center of Excellence, University of Antwerp, B-2020 Antwerp, Belgium

${ }^{4}$ Université Paris-Saclay, CNRS, Centre de Nanosciences et de Nanotechnologies, 91120, Palaiseau, France.

${ }^{5}$ ONERA - The French Aerospace Lab, 6, chemin de la Vauve aux Granges, Palaiseau, BP 80100,91123 , France

${ }^{6}$ Université de Lille, CNRS, Centrale Lille, ISEN, Université de Valenciennes, UMR 8520-IEMN, 59000 Lille, France.

To whom correspondence should be sent: Ihuillier@insp.upmc.fr

\section{Table of content}

1. Nanocrystal synthesis

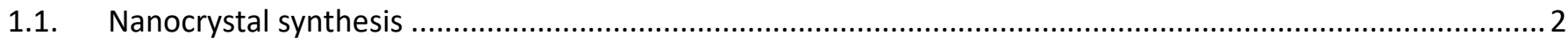

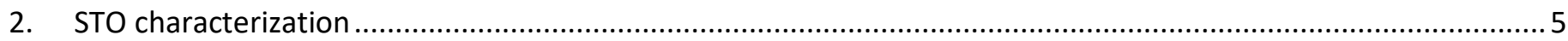

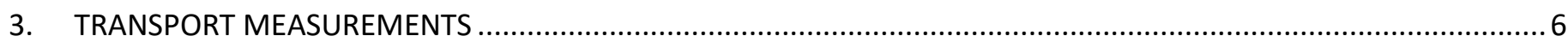

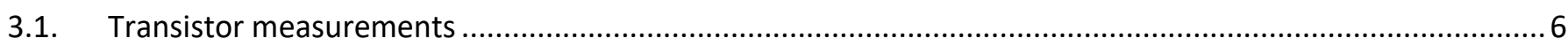

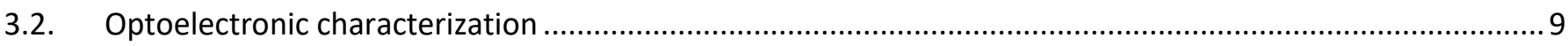

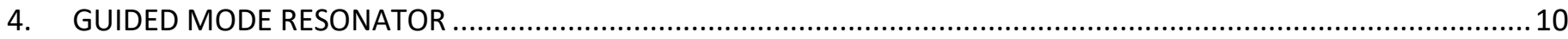

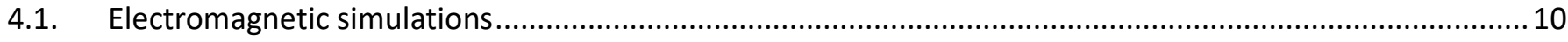

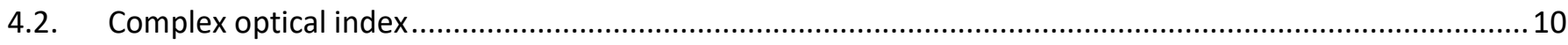

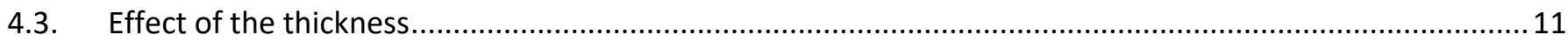

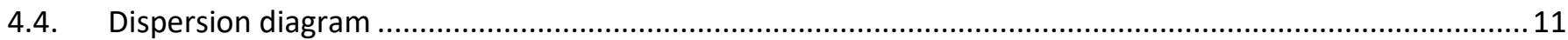

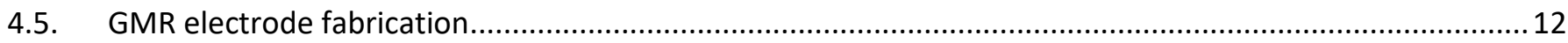

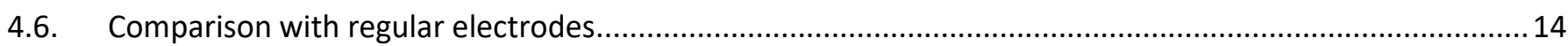

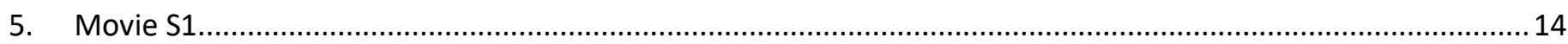

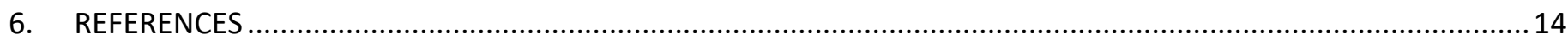




\section{Nanocrystal synthesis}

\subsection{Nanocrystal synthesis}

HgTe CQD synthesis with band edge at $2000 \mathrm{~cm}^{-1}$ : The reaction is taken from Keuleyan et al. ${ }^{1}$ The absorption spectrum shows a band-edge around $2000 \mathrm{~cm}^{-1}$, see Figure S 1a. The particles have a tetrahedron shape with a typical size around $12 \mathrm{~nm}$, see Figure $\mathrm{S} 1 \mathrm{~b}$.
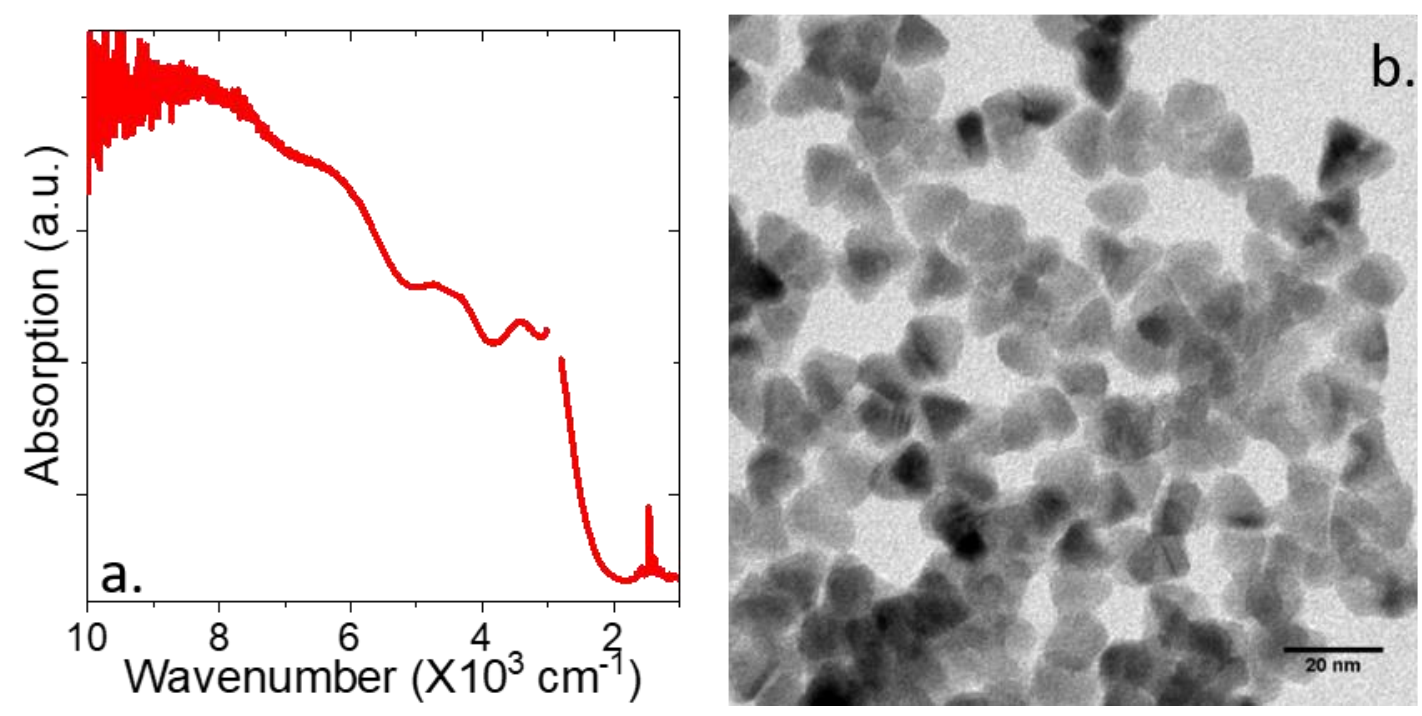

Figure $S 1$ a. Absorption spectrum for the HgTe CQDs with a band-edge at $2000 \mathrm{~cm}^{-1}$. b. Transmission Electron Microscopy image for HgTe CQDs with a band-edge at $2000 \mathrm{~cm}^{-1}$.

HgTe CQD synthesis with band edge at $4000 \mathrm{~cm}^{-1}$ : In a $100 \mathrm{~mL}$ three neck flask, $543 \mathrm{mg}$ of $\mathrm{HgCl}_{2}$ and $50 \mathrm{~mL}$ of oleylamine was degassed under vacuum at $110^{\circ} \mathrm{C}$. Meanwhile, $2 \mathrm{~mL}$ of TOP:Te (1 M) were extracted from the glove box and mixed with $8 \mathrm{~mL}$ of oleylamine. After the atmosphere was switched to $\mathrm{N}_{2}$ and the temperature stabilized at $82{ }^{\circ} \mathrm{C}$, the TOP:Te solution was quickly injected. After $2 \mathrm{~min} 30 \mathrm{~s}, 10 \mathrm{~mL}$ of a mixture of DDT in toluene (10\% of DDT) was injected and a water bath was used to quickly decrease quickly the temperature. The content of the flask was split over 4 falcons and $\mathrm{MeOH}$ was added. After centrifugation, the formed pellet was redispersed in one falcon with toluene. The solution was precipitated a second time using ethanol. Again, the formed pellet was redispersed in toluene. At this step the nanocrystals were centrifuged in pure toluene to get rids of the lamellar phase. The solid phase was discarded. The stable phase was transferred in a weighted falcon and finally precipitated using methanol. The solid was dried under vacuum for $30 \mathrm{~min}$ (in glove box vacuum chamber). Finally, nanocrystals were redispersed with a $50 \mathrm{mg} \cdot \mathrm{mL}^{-1}$ concentration in toluene. The absorption spectrum shows a band-edge around $4000 \mathrm{~cm}^{-1}$, see Figure $\mathrm{S} 2 \mathrm{a}$. The particles have a multi-pod shape according to TEM image in Figure S $2 \mathrm{~b}$. 

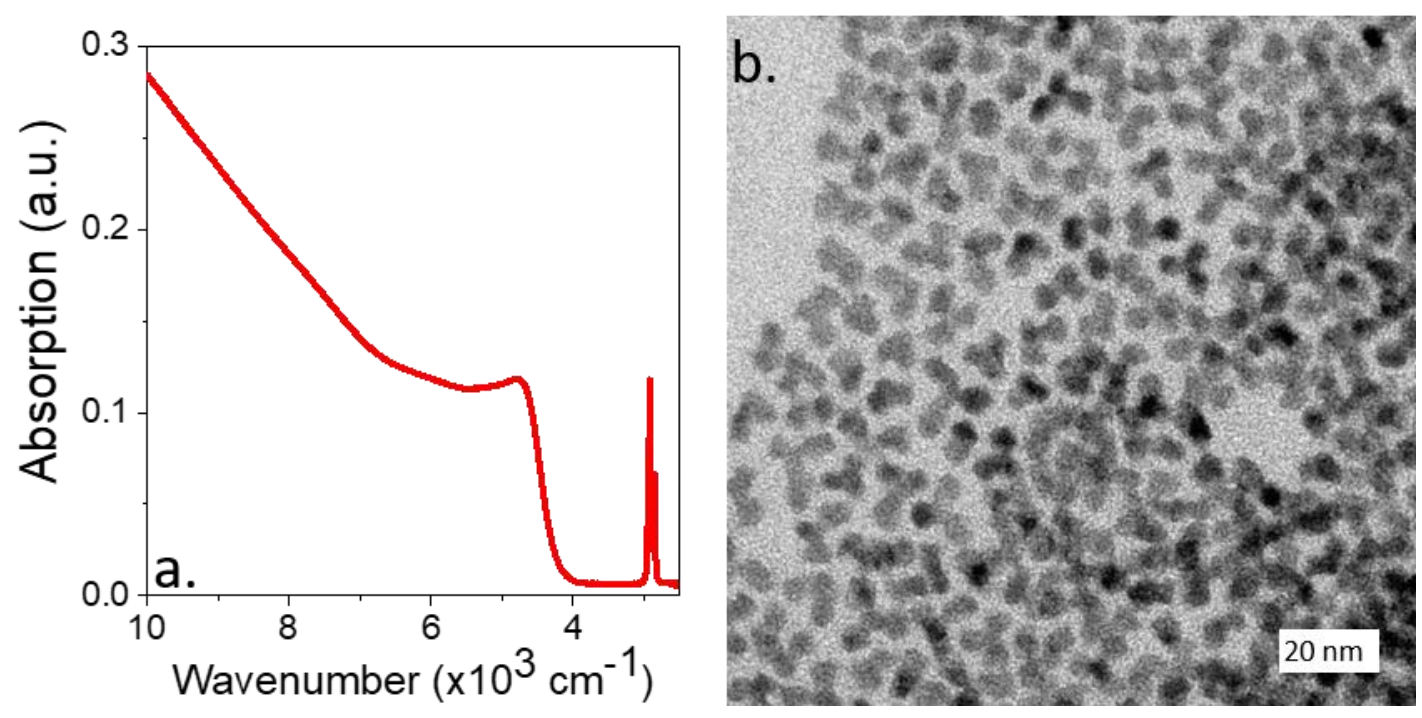

Figure S 2 a. Absorption spectrum for HgTe CQDs with a band-edge at $4000 \mathrm{~cm}^{-1}$. b. Transmission Electron Microscopy image for HgTe CQDs with a band-edge at $4000 \mathrm{~cm}^{-1}$.

HgTe CQD synthesis with band edge at $6500 \mathrm{~cm}^{-1}$ : In a $100 \mathrm{~mL}$ three neck flask, $540 \mathrm{mg}$ of $\mathrm{HgCl}_{2}(2 \mathrm{mmol})$ and $50 \mathrm{~mL}$ of oleylamine were degassed under vacuum at $110{ }^{\circ} \mathrm{C}$. Meanwhile $2 \mathrm{~mL}$ of TOP:Te $(1 \mathrm{M})$ were extracted from glove box and mixed with $8 \mathrm{~mL}$ of oleylamine. After the atmosphere was switched to $\mathrm{N}_{2}$ and the temperature stabilized at $56{ }^{\circ} \mathrm{C}$, the pre-heated TOP:Te solution was quickly injected. After $3 \mathrm{~min}, 10 \mathrm{~mL}$ of a mixture of DDT in toluene ( $20 \%$ of DDT) were injected and a water bath was used to quickly decrease quickly the temperature. The content of the flask was splitted over 4 falcons and $\mathrm{MeOH}$ was added. After centrifugation the formed pellet was redispersed in one falcon with toluene. The solution was precipitated a second time with absolute $\mathrm{EtOH}$. Again, the formed pellet was redispersed in toluene. At this step the nanocrystals were centrifuged in pure toluene to get rids of the lamellar phase. The solid phase was discarded. The stable phase was transferred in a weighted falcon and finally precipitated using $\mathrm{MeOH}$. The solid was dried under vacuum for $1 \mathrm{~h}$ (in glove box vacuum chamber). Finally, nanocrystals were redispersed with a $50 \mathrm{mg} \cdot \mathrm{mL}^{-1}$ concentration in toluene and filtrated. The absorption spectrum shows a band-edge around $6500 \mathrm{~cm}^{-1}$, see Figure $S 3$ a. The particles have a rod shape with a typical size around $6 \mathrm{~nm}$, see Figure $S 3 b$.
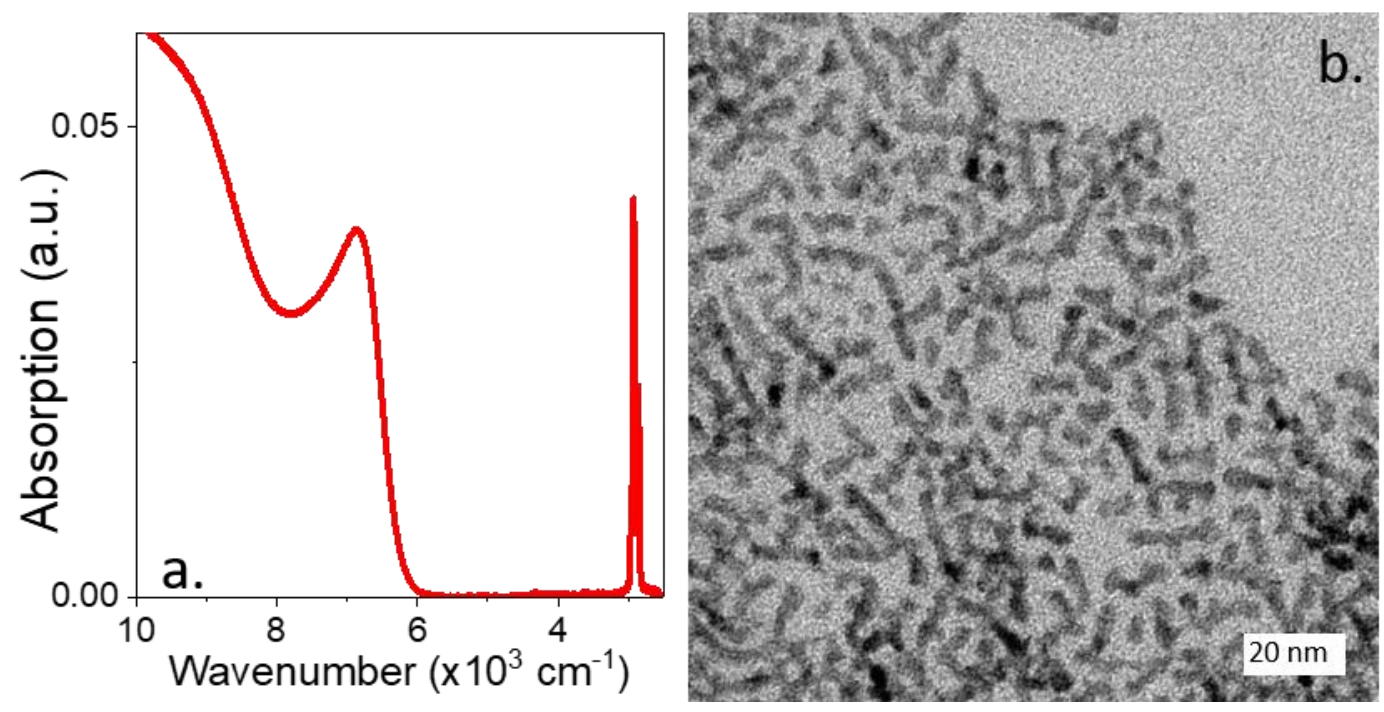

Figure S 3 a. Absorption spectrum for HgTe CQDs with a band-edge at $6500 \mathrm{~cm}^{-1} . b$. Transmission Electron Microscopy image for HgTe CQDs with a band-edge at $6500 \mathrm{~cm}^{-1}$. 
PbS CQD Synthesis with band edge at $6500 \mathrm{~cm}^{-1}$ : In a three-neck flask, $300 \mathrm{mg}$ of $\mathrm{PbCl}_{2}$ and $7.5 \mathrm{~mL}$ of OLA are degassed, first at room temperature and then at $110^{\circ} \mathrm{C}$ for $30 \mathrm{~min}$. Meanwhile $30 \mathrm{mg}$ of $\mathrm{S}$ powder is mixed with $7.5 \mathrm{~mL}$ of OLA until full dissolution and an orange clear solution is obtained. Then under $\mathrm{N}_{2}$ at $160^{\circ} \mathrm{C}$, this solution of $\mathrm{S}$ is quickly added to the flask. After 15 minutes, the reaction is quickly quenched by addition of $1 \mathrm{~mL}$ of oleic acid and $10 \mathrm{~mL}$ of hexane. The nanocrystals are precipitated with ethanol and redispersed in toluene. This washing step is repeated one more time. The solution is then centrifuged to remove the unstable phase. The supernatant is precipitated with methanol and redispersed in toluene. Finally, the PbS CQD solution in toluene is filtered through a $0.2 \mu \mathrm{m}$ PTFE filter. The obtained solution is used for further characterization and device fabrication. The absorption spectrum shows a bandedge around $6500 \mathrm{~cm}^{-1}$, see Figure $S 4$ a. The particles have a spherical shape with a typical size around $8 \mathrm{~nm}$, see Figure $S 4 b$.
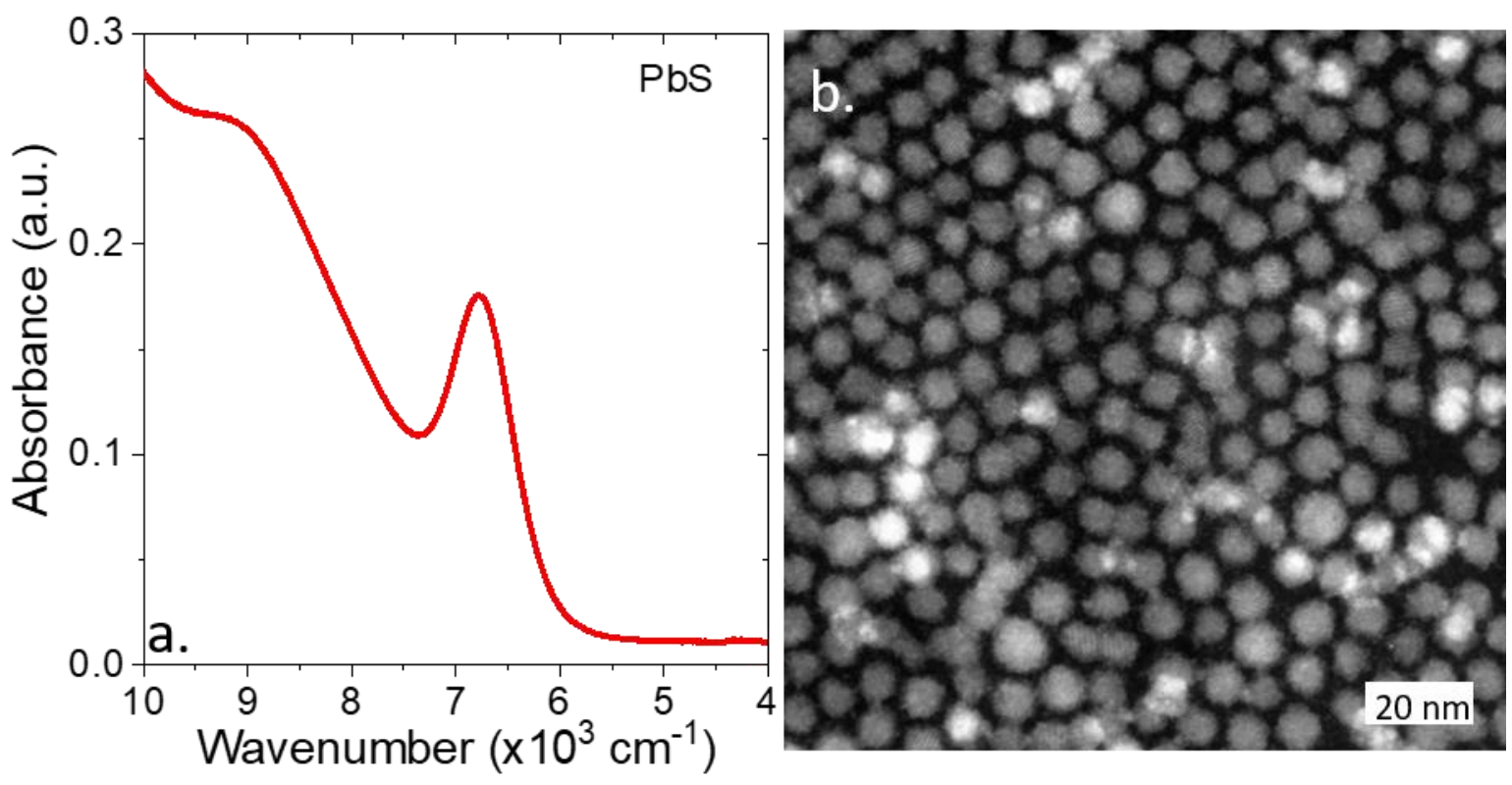

Figure $S 4$ a. Absorption spectrum for the PbS CQDs with a band-edge at $6500 \mathrm{~cm}^{-1}$. b. High Angle Annular Dark Field Scanning Transmission Electron Microscopy (HAADF-STEM) image for the PbS CQDs with a band-edge at $6500 \mathrm{~cm}^{-1}$. 


\section{STO characterization}

We have conducted AFM measurement on the STO substrate to check its surface quality, see Figure S 5 . The RMS roughness is estimated to be $1.4 \mathrm{~nm}$ which is smaller than the NC size.
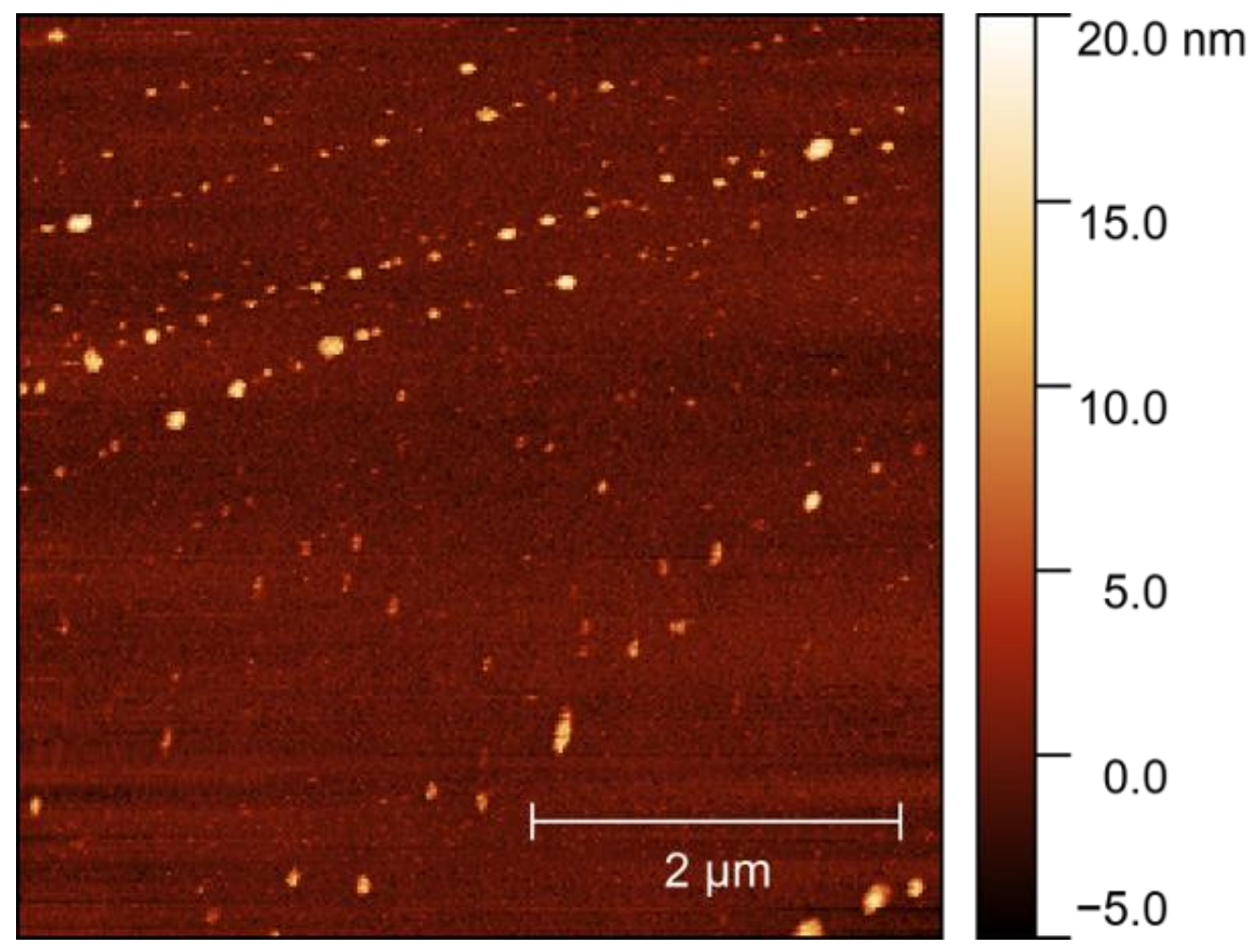

Figure S 5 AFM image of a pristine STO substrate 


\section{TRANSPORT MEASUREMENTS}

\subsection{Transistor measurements}

- Using STO ferroelectric gate

Electrodes fabrication for transistor. A polished STO substrate is first rinsed and then sonicated in acetone for $5 \mathrm{~min}$. The wafer is then rinsed with acetone and isopropanol and finally placed in a $\mathrm{O}_{2}$ plasma cleaner for 5 min. Then $\mathrm{TI}$ Prime is spin coated on the substrate and baled at $120^{\circ} \mathrm{C}$ for $2 \mathrm{~min}$ to promote adhesion of the resist. AZ 5214E resist is spin coated on the substrate and baked at $110^{\circ} \mathrm{C}$ for $90 \mathrm{~s}$. The resist is exposed through a shadow mask to UV illumination for $1.5 \mathrm{~s}$. The resist is baked again at $127^{\circ} \mathrm{C}$ for $2 \mathrm{~min}$ and re-exposed to UV (without the mask) for $40 \mathrm{~s}$. The resist is then developed using AZ726 MIF. The film is rinsed with water and finally dried. The remaining organic layer is removed by plasma cleaning. $5 \mathrm{~nm}$ of chromium and $80 \mathrm{~nm}$ of gold are thermally evaporated and the remaining resist is removed by an acetone lift-off during one hour. The electrodes are then rinsed with isopropanol and finally dried. The interdigitated electrodes include 25 digits, each $2.5 \mathrm{~mm}$ long with a $10 \mu \mathrm{m}$ gap between them. The optical area of the device is $0.037 \mathrm{~cm}^{2}$.

DC Transport: The sample is connected to a Keithley 2634b, which controls the drain bias $\left(V_{D S}\right)$ and measures the associated current $\left(\mathrm{IDS}_{\mathrm{DS}}\right)$. This measure is carried out in the dark or under illumination using $1.55 \mu \mathrm{m}$ laser diode.

Transistor Measurement: The sample is connected to the two channels of a Keithley 2634b, which sets the drain source bias $\left(\mathrm{V}_{\mathrm{DS}}\right)$, controls the gate bias $\left(\mathrm{V}_{\mathrm{GS}}\right)$ and measure the associated currents (IDS and $\left.\mathrm{I}_{G S}\right)$.
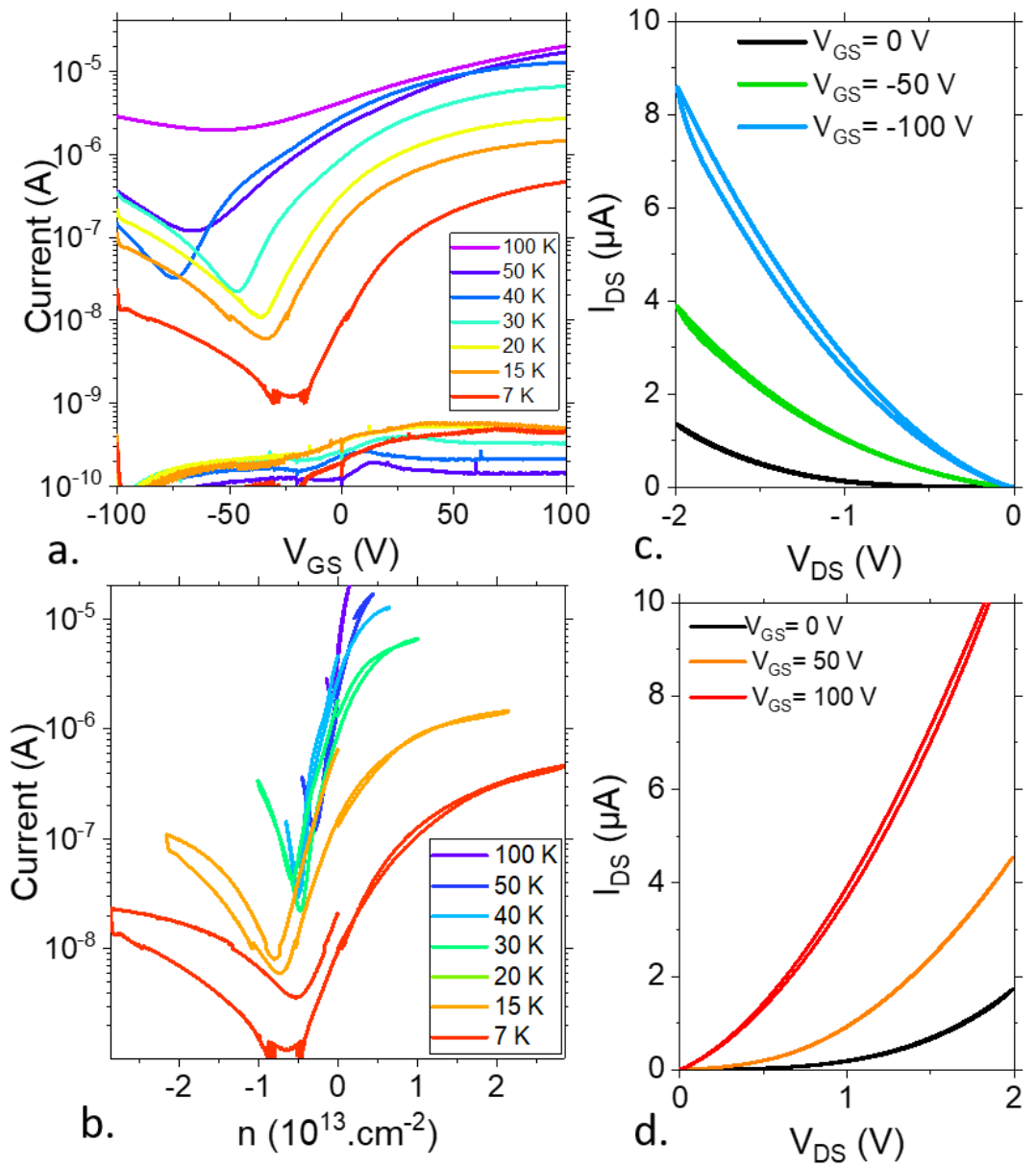

Figure $S 6$ a. Transfer curves for a transistor which channel is made of HgTe 2k CQDs and for which the gate is made of a STO substrate. The drain source bias is set to $1 \mathrm{~V}$. b. Drain current as a function of carrier density for a transistor which channel is made of HgTe $2 k$ CQDs and for which the gate is made of a STO substrate. 
c. I-V curves for the same device under hole injection condition. d. I-V curves for the same device under electron injection condition.

To demonstrate the generality of the gating method, we apply to HgTe CQDs with other band edge, as well as for $\mathrm{PbS}$ CQDs. In all case we have obtained a strong current modulation, see Figure $\mathrm{S} 7$.

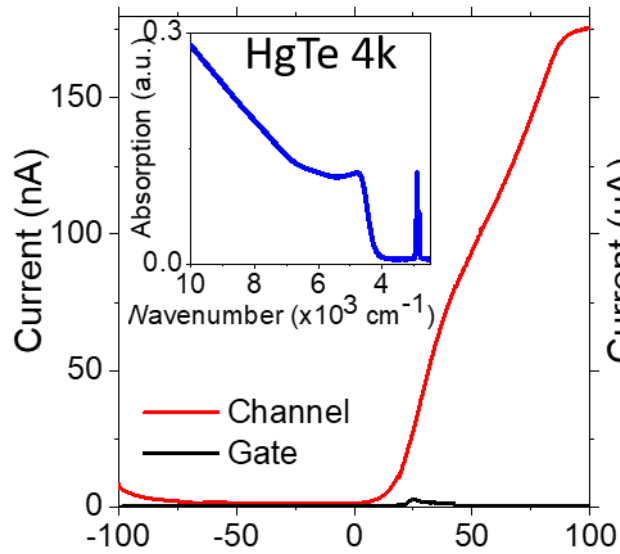

a. Gate voltage (V)

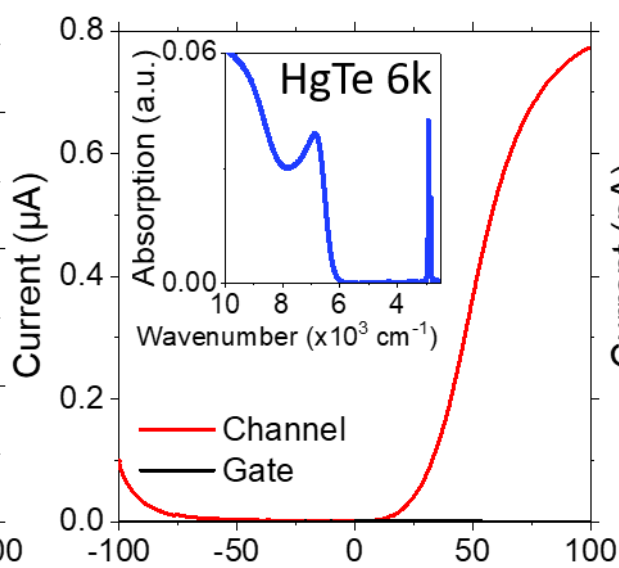

b. $\quad$ Gate voltage $(\mathrm{V})$

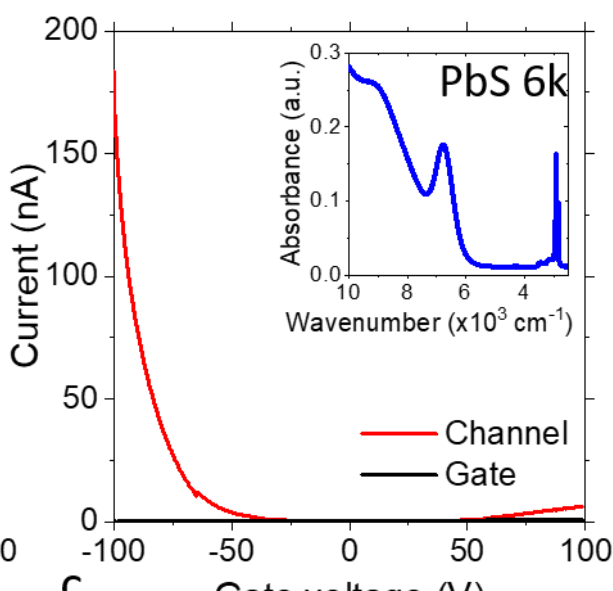

C. $\quad$ Gate voltage $(\mathrm{V})$

Figure S 7 a. Transfer curves for a FET which gate is made of a STO substrate while the channel is an array of HgTe 4k CQDs. b. Transfer curves for a FET which gate is made of a STO substrate while the channel is an array of HgTe 6k CQDs. c Transfer curves for a FET which gate is made of a STO substrate while the channel is an array of PbS $6 \mathrm{k} C Q D s$. Measurements are made at $30 \mathrm{~K}$

\section{- Other gating methods}

Electrolyte gating. For electrolyte gating we first mixed in a glove box $0.5 \mathrm{~g}$ of $\mathrm{LiClO}_{4}$ with $2.3 \mathrm{~g}$ of PEG $\left(\mathrm{MW}_{\mathrm{W}}=6\right.$ $\left.\mathrm{kg} \cdot \mathrm{mol}^{-1}\right)$. The vial is heated at $170^{\circ} \mathrm{C}$ on a hot plate for $2 \mathrm{~h}$ until the solution turns clear. The electrolyte solution is warmed to around $100{ }^{\circ} \mathrm{C}$ and brushed on the top of the NC film.

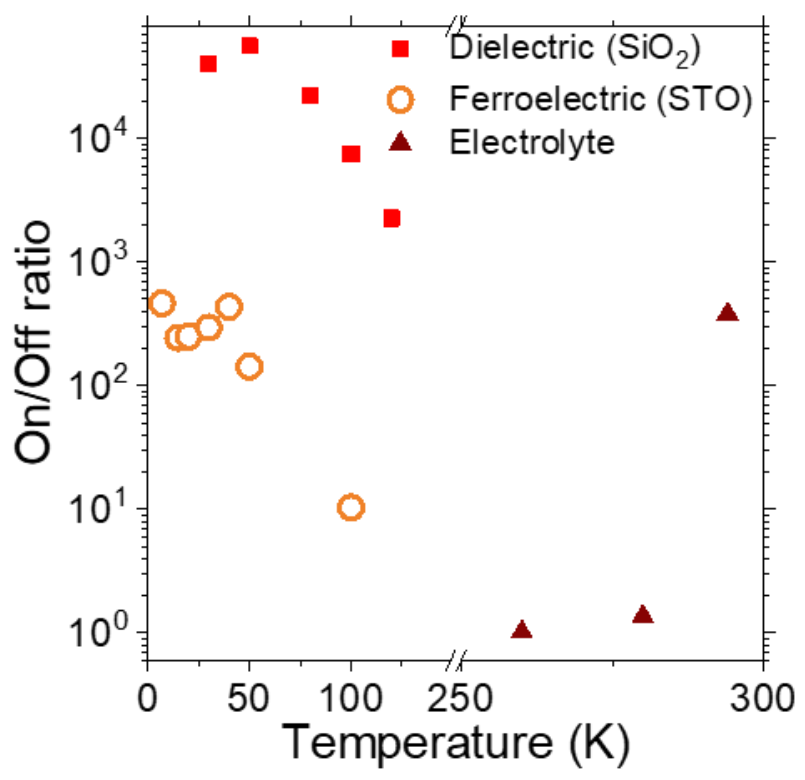

Figure $S 8$ on/off ratio from the FET transfer curve as a function of the temperature for the different gates. 

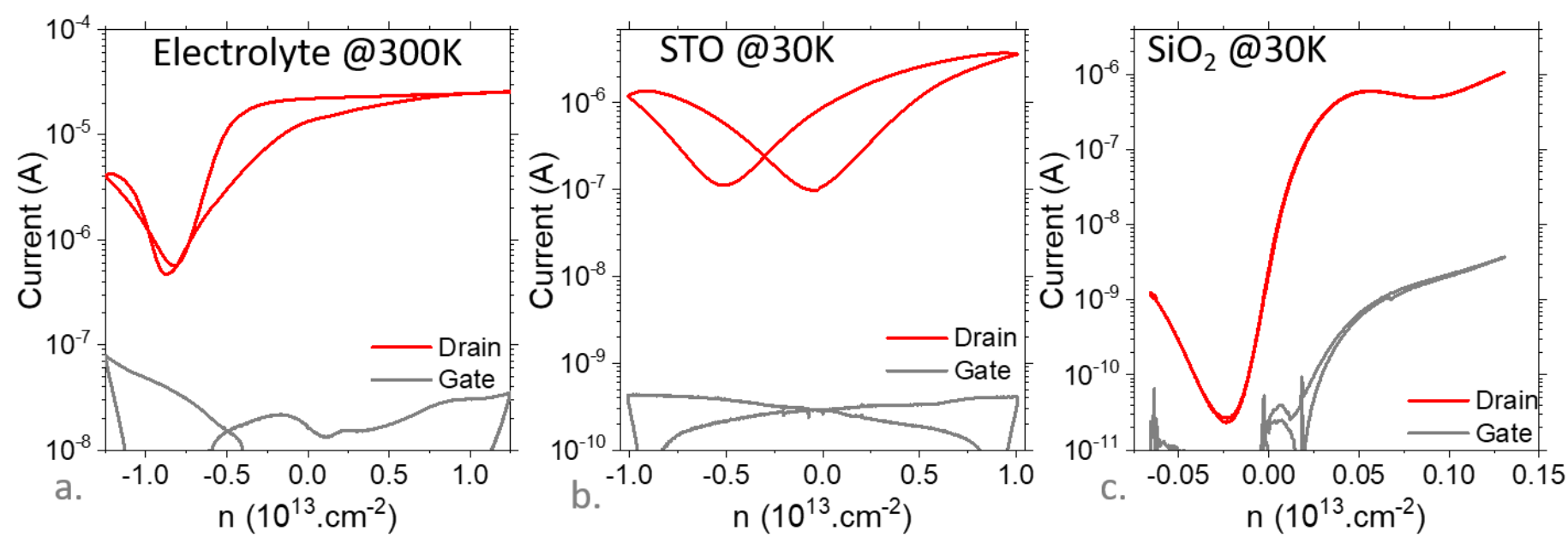

Figure $S 9$ a-c are respectively the drain and gate current as a function of the film carrier density for electrolyte $(T=300 \mathrm{~K})$, STO $(\mathrm{T}=30 \mathrm{~K})$ and $\mathrm{SiO}_{2}(\mathrm{~T}=30 \mathrm{~K})$ gate based FET which channel is made of HgTe $2 \mathrm{k} \mathrm{NCS}$.

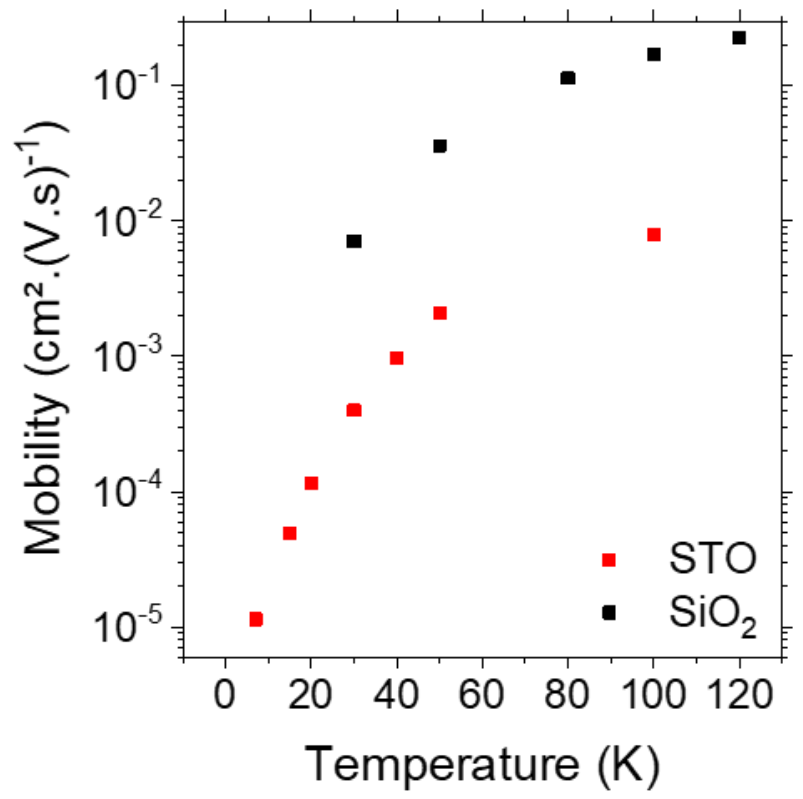

Figure $S 10$ Electron mobility from the FET transfer curves as a function of the temperature, for ferroelectric gate (ie STO) and for dielectric gate (ie $\mathrm{SiO}_{2}$ ). 


\subsection{Optoelectronic characterization}

Responsivity measurement: A $1.55 \mu \mathrm{m}$ laser is chopped at $1 \mathrm{kHz}$ and shone on the sample. The drain-source and the gate-source voltages are set by a Keithley K2634b. A DLPCA transimpedance amplifier is connected on the drain branch of the circuit to amplify the current before sending it to a MFLI lock-in amplifier from Zurich instrument. The photocurrent is then acquired of the lock-in. The power of the laser is measured using a Thorlabs PM100A power meter equipped with a wide range detector. A scheme of the setup is given in Figure S 11.

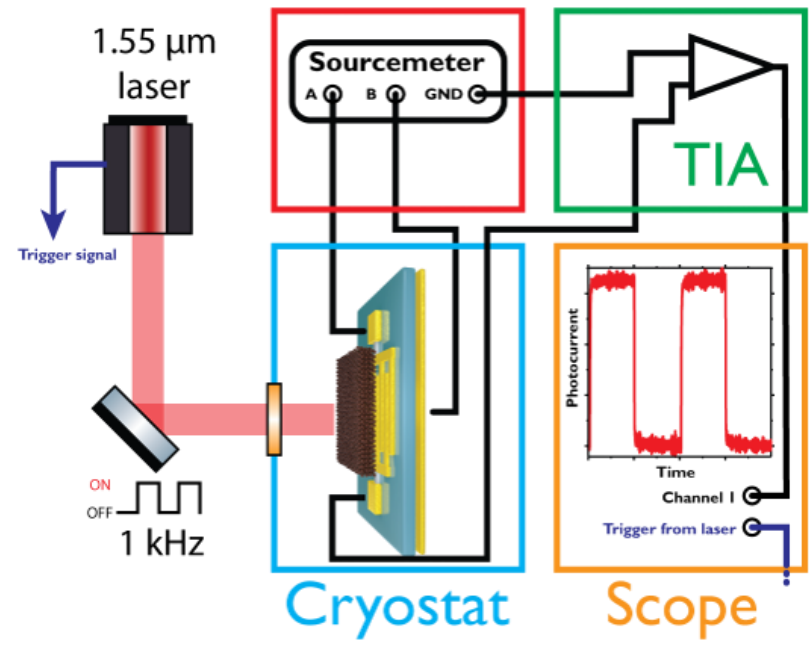

Figure $S 11$ Scheme of the experimental setup used to determine the responsivity.

Spectral response measurement: We use a thermo Fischer FTIR as interferometer. The globar source of the FTIR is fed into the interferometer. A XT-KBr beamsplitter is used and the light finally shone on the sample. The sample is biased using the bias supply of a Femto DLPCA-200 amplifier. The current is then amplified by a Femto DLPCA-200, and fed back to the electronic of the FTIR. A scheme of the setup is given in Figure $\mathrm{S} 12$.

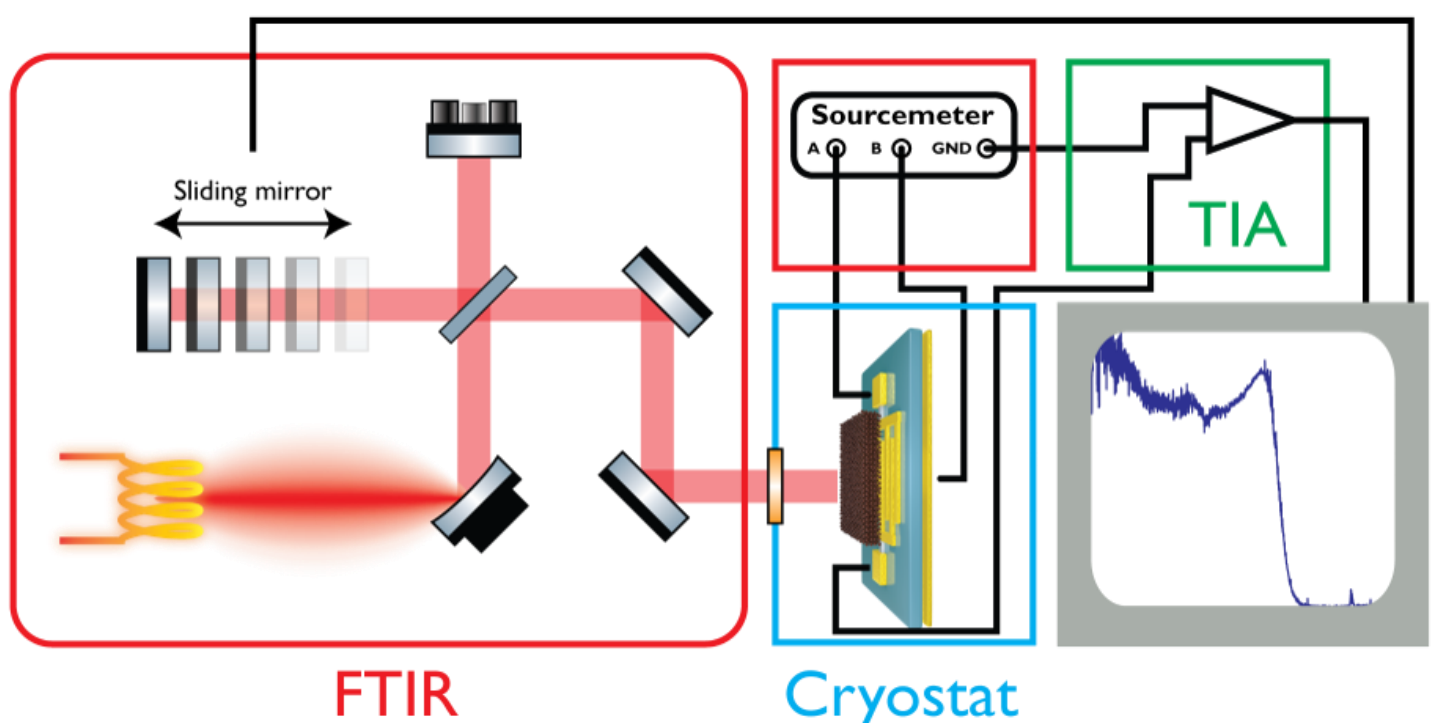

Figure S 12 Scheme of the experimental setup used to determine the spectral response.

Noise measurement: The sample is biased (at $1 \mathrm{~V}$ bias, kept in the dark) using the bias supply of a Femto DLPCA-200 amplifier. The current is then amplified by a Femto DLPCA-200, and fed into a SRS SR780 signal analyzer.

Detectivity determination. The detectivity (in Jones) of the sample is determined using the formula: $D^{*}=\frac{R \sqrt{A}}{S_{I}}$, where $\mathrm{R}\left(\right.$ in $\left.\mathrm{A} . \mathrm{W}^{-1}\right)$ is the responsivity, $\mathrm{S}$ is the noise $(\mathrm{A} / \sqrt{\mathrm{Hz}})$ and $\mathrm{A}$ the area of the device $\left(\mathrm{cm}^{2}\right)$. 


\section{GUIDED MODE RESONATOR}

\subsection{Electromagnetic simulations}

For the GMR device, the layers are given in Table S 1: Layer thicknesses for each stack.

\begin{tabular}{cc}
\hline \hline Layer & Interdigitated on Au/SiO ${ }_{2}$ for GMR \\
\hline $\begin{array}{c}\text { External } \\
\text { environment }\end{array}$ & Air \\
\hline Film & $\mathrm{HgTe}$ \\
$\mathrm{d}=310 \mathrm{~nm}$ \\
\hline Structure & $\mathrm{HgTe} / \mathrm{Au}\left(\right.$ period $\mathrm{p}$ and $\left.\mathrm{w}_{\mathrm{Au}}\right)$ \\
& $\mathrm{t}_{\mathrm{g}}=50 \mathrm{~nm}$ \\
\hline Substrate & $\mathrm{STO}$ \\
& $200 \mathrm{~nm}$ \\
\hline \hline
\end{tabular}

Table S 1: Layer thicknesses for each stack

The absorption map is deduced from the electric field at each point:

$$
\begin{gathered}
\text { Absorption }=\frac{\pi}{\lambda} \int_{S} \operatorname{Im}(\varepsilon(M))\left(\left|E_{y}\right|^{2}\right) d S, \quad \text { In TE polarisation } \\
\text { Absorption }=\frac{\pi}{\lambda} \int_{S} \operatorname{Im}(\varepsilon(M))\left(\left|E_{x}\right|^{2}+\left|E_{z}\right|^{2}\right) d S, \quad \text { In TM polarisation }
\end{gathered}
$$

\subsection{Complex optical index}

The last input needed for these calculations is the complex refractive index of each layer. For the gold ${ }^{2}$ and for $\mathrm{STO}^{3,4}$ we used previously reported refractive indices. For the real part of the nanocrystal film refractive index, the value of 1.9 is commonly used for nanocrystal thin film. Figure $\mathrm{S} 13$ shows the effect of the real part of the HgTe NCs film optical index. The lower the optical index, the bluer the absorption.

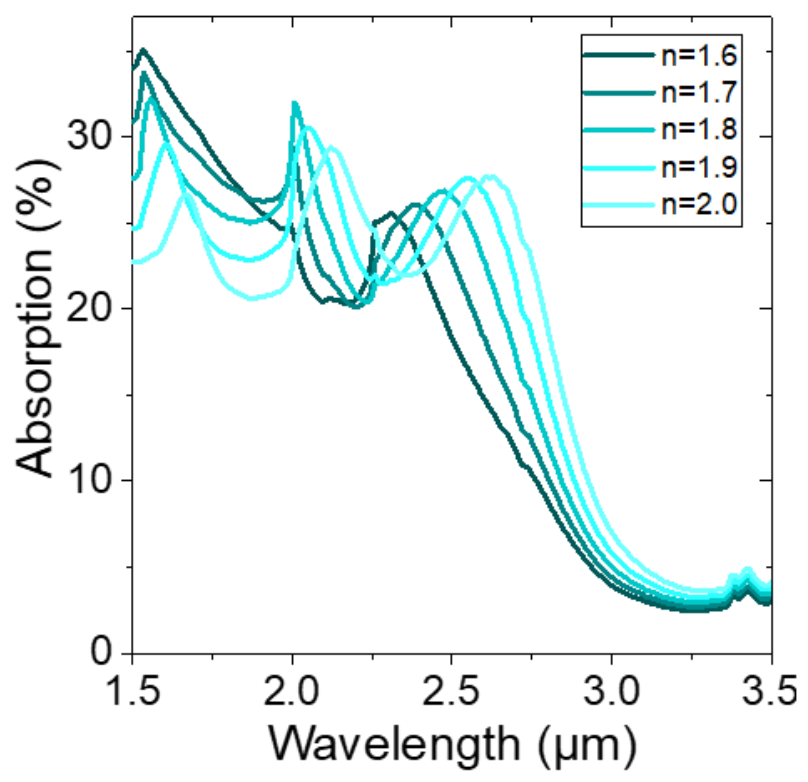

Figure S 13 Effect of the real part of the HgTe NC film optical index on the GMR absorption spectrum. 
For $\mathrm{HgTe}$, the absorption coefficient is measured to be $3 \times 10^{3} \mathrm{~cm}^{-1}$ which give $\mathrm{k}=0.04$ at the band edge. The wavelength dependence of $\mathrm{k}$ is given in Figure $\mathrm{S} 14$.

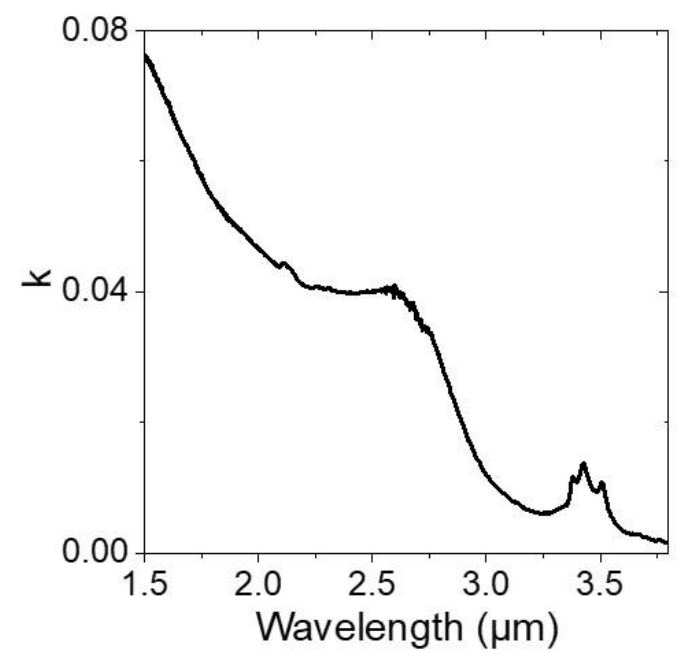

Figure $\mathrm{S} 14$ Imaginary part of the refractive index of $\mathrm{HgTe} 4 \mathrm{k}$

\subsection{Effect of the thickness}

In order to look at the robustness of the geometrical parameter, we have quantized how the TM spectra is affected by the continuous film thickness (Figure S 15).

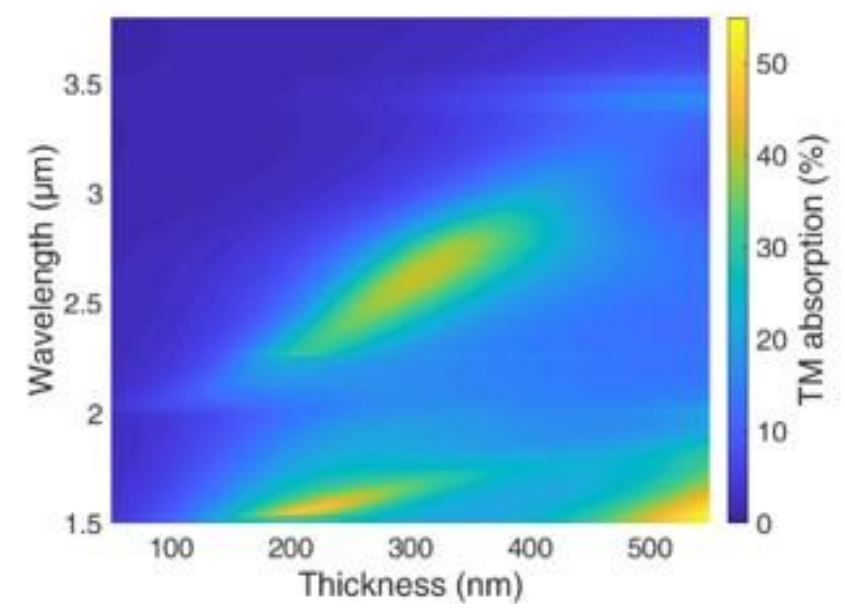

Figure S 15 Absorption spectrum as a function of the thickness of the film.

\subsection{Dispersion diagram}

The dispersion diagram of the TM (reps TE) component is given Figure $\mathrm{S} 16$ a (resp b.). On the $y$-axis, we give the incoming wavenumber $(\sigma)$ and on the $x$-axis, we give the $x$-component of the incoming wavevector. $k_{x}$ depends on incidence angle $\theta$ and on wavenumber $\sigma: k_{x}=2 \pi \cdot \sigma \cdot \sin (\theta)$. The GMR peaks are highlighted with pink dashed line. The grey dashed line (in the TE map) is the limit of diffraction order appearing in air. The white dotted line corresponds to the limit of diffraction order appearing in STO or NC film. 


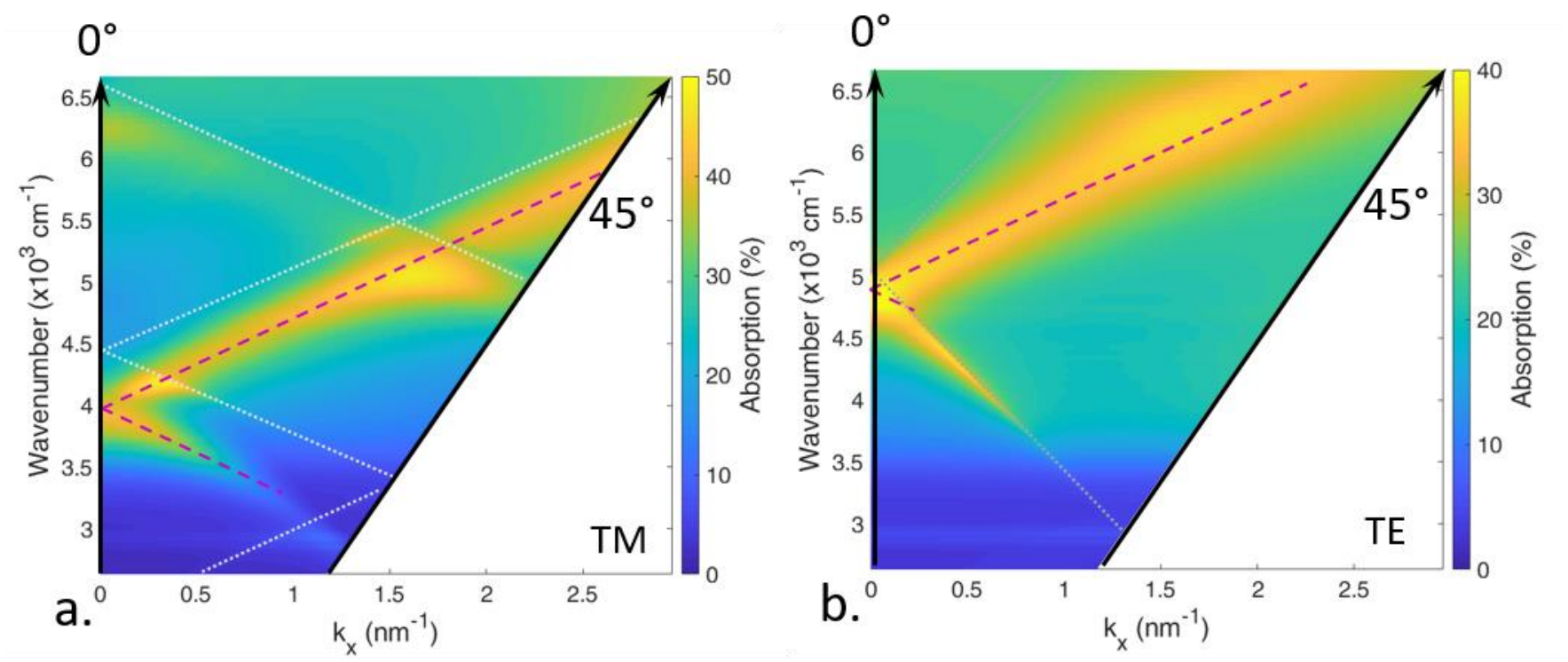

Figure S 16 Dispersion diagram for the TM (left) and the TE (right) componant of the GMR structure. Pink dashed line correponds to GMR peaks. Dotted lines correponds to the limits of diffraction order appearing in air, STO or HgTe NC film.

\subsection{GMR electrode fabrication}

The Figure $\mathrm{S} 17$ is a schematic of these main fabrication steps.

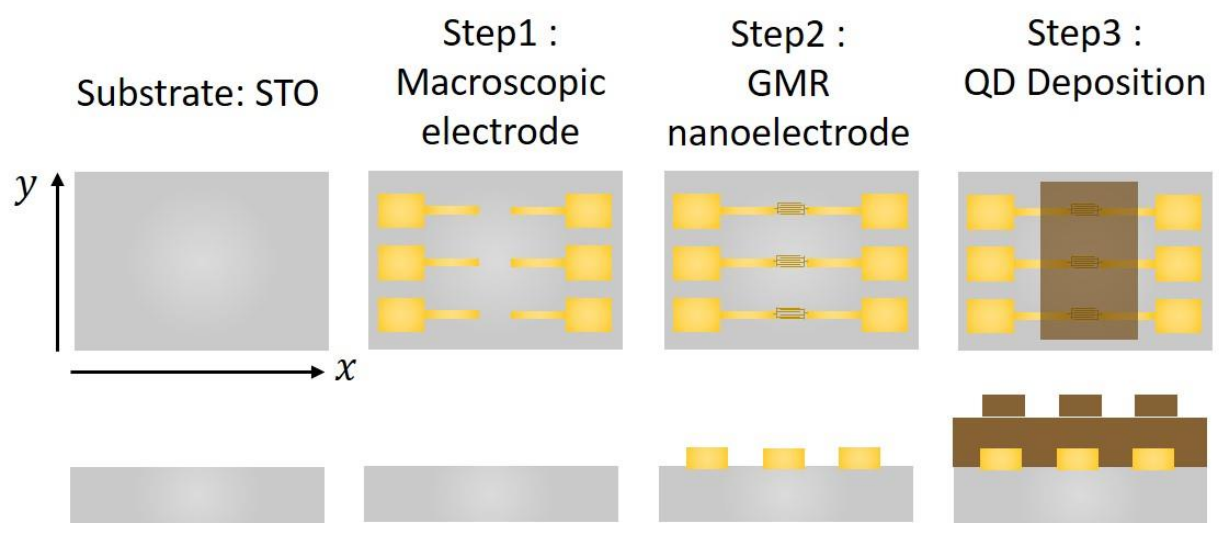

Figure S 17 Main fabrication steps of the electrodes used to induce a GMR. Top part is a top view, while bottom part is a side view. Step one relates to the deposition of the macroscopic electrodes. Step 2 relates to the fabrication of the interdigitated electrodes inducing GMR with e-beam lithography.. Step 3 relates to the deposition of the film deposition. ${ }^{5}$.

Electrode inducing GMR. These electrodes are made using 4 different steps.

Step 0: Substrate preparation. On the back side of the substrate a layer of $\mathrm{Cr} / \mathrm{Au}(5 / 80 \mathrm{~nm})$ is evaporated.

Step 1: Deposition of macroscopic pads for reporting electric contacts. The surface of the STO substrate is cleaned by sonication in acetone. The wafer is rinsed with acetone, then isopropanol and dried with a $\mathrm{N}_{2}$ gun. A final cleaning is made using an $\mathrm{O}_{2}$ plasma. An adhesion promoter (TI PRIME) is spin-coated onto the substrate and baked at $120^{\circ} \mathrm{C}$ for $2 \mathrm{~min}$. AZ5214E is spin-coated and baked at $110^{\circ} \mathrm{C}$ for $90 \mathrm{~s}$. The substrate is exposed under UV through a pattern mask for $1.5 \mathrm{~s}$. The film is then baked at $127^{\circ} \mathrm{C}$ for 2 min in order to 
invert the resist. Then a $40 \mathrm{~s}$ flood exposure is performed. The resist is developed using a bath of AZ726 for $32 \mathrm{~s}$, rinsed in pure water and finally dried with $\mathrm{N}_{2}$. We then deposit $5 \mathrm{~nm}$ of chromium and $80 \mathrm{~nm}$ of gold using thermal evaporation. The lift-off is performed by dipping the film in acetone for 1 hour. The gold macroscopic electrode is rinsed using isopropanol and dried with a $\mathrm{N}_{2}$ flow.

Step 2: Deposition of nanoelectrodes. After having rinsed the sample with acetone and isopropanol, a layer of A6 PMMA 950 is spin-coated onto the substrate and baked for $15 \mathrm{~min}$ at $150{ }^{\circ} \mathrm{C}$. A layer of Electra 96 is spin-coated onto the PMMA layer and backed at $90{ }^{\circ} \mathrm{C}$ for two minutes. The samples are transferred in a Zeiss Supra 40 SEM with Raith elphy quantum device for electron beam lithography. The operating bias is set to $20 \mathrm{kV}$ and the aperture to $15 \mu \mathrm{m}$. The current is measure at $70 \mathrm{pA}$. The dose is set at $220 \mu \mathrm{C} . \mathrm{cm}^{-2}$. The electra layer is removed by dipping the film in water for $30 \mathrm{~s}$. The PMMA is developed by dipping the film in a solution of MIBK:isopropanol (1:3) for $50 \mathrm{~s}$ and rinsed in pure isopropanol for $20 \mathrm{~s}$. We then deposit a 5 $\mathrm{nm}$ layer of chromium and a $50 \mathrm{~nm}$ layer of gold using thermal evaporation. The lift-off is performed by dipping the film in acetone overnight. Sonication at low power is necessary to remove the remaining resist. 20 pairs of digits are deposited as electrodes. The electrodes are $500 \mu \mathrm{m}$ long. For a resonance at $2.3 \mu \mathrm{m}$ the digits are $1050 \mathrm{~nm}$ wide and separated by $1050 \mathrm{~nm}$.

Step 3 QD film deposition. The film of QD is deposited by spin-coating from an ink as described in 1.4, spincoating parameters are $1300 \mathrm{rpm}$ for $200 \mathrm{~s}$ and $2500 \mathrm{rpm}$ for $120 \mathrm{~s}$. The film is $310 \mathrm{~nm}$ thick. 


\subsection{Comparison with regular electrodes}

Figure $\mathrm{S} 18$ provides the dark current and responsivity for the regular electrodes (ie without GMR) for a phototransistor based on STO gate and $310 \mathrm{~nm} \mathrm{HgTe} \mathrm{NC} \mathrm{film} \mathrm{as} \mathrm{channel.}$

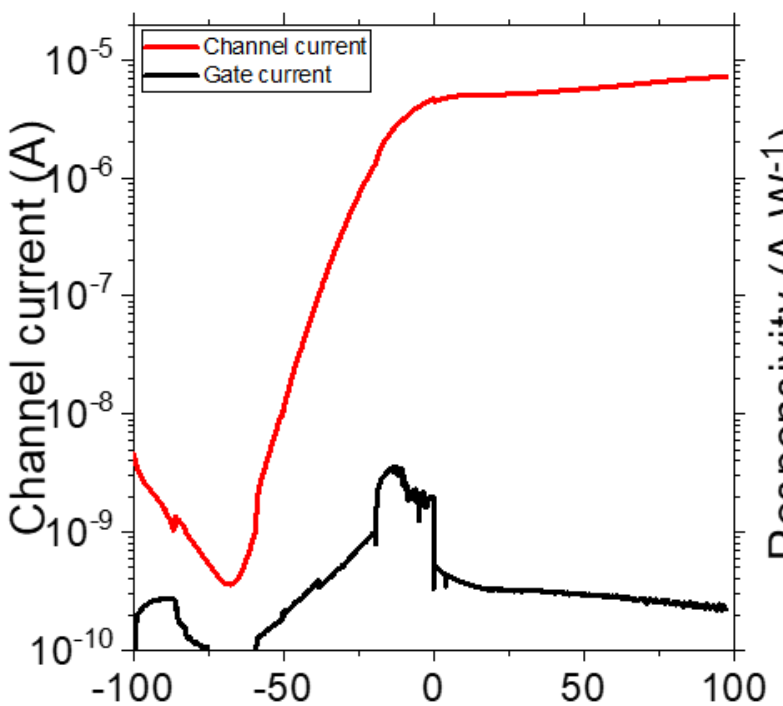

a. Gate voltage (V)

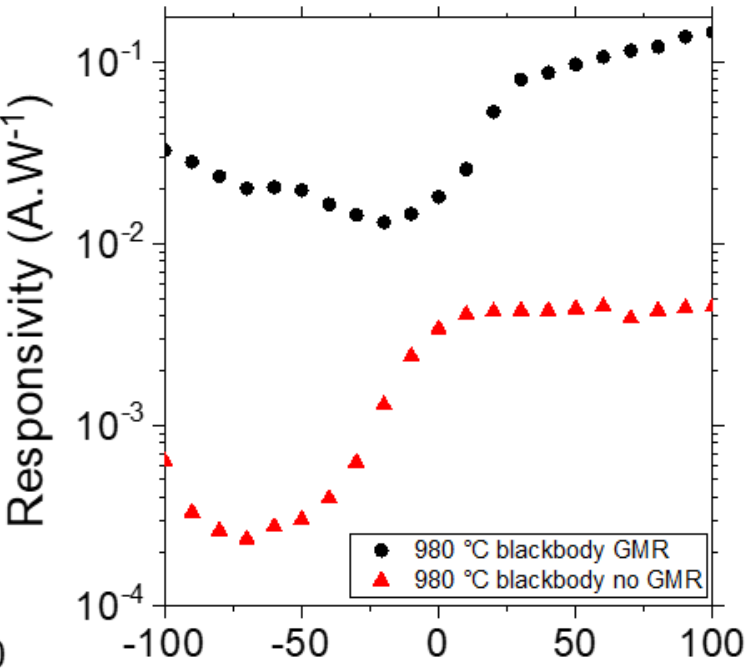

b. Gate Voltage (V)

Figure S 18a Transfer curves for HgTe NCs film onto STO subtrate without GMR. $b$. Responsivity as a fuction of the applied gate bias for an HgTe NC film onto a STO substrate ( $\approx 300 \mathrm{~nm}$ as thickness) for the regular and GMR electrodes.

\section{Movie S1}

Movie S1 is a 3D view of the nanocrystal shown in figure 1

\section{REFERENCES}

(1) Keuleyan, S.; Lhuillier, E.; Guyot-Sionnest, P. Synthesis of Colloidal HgTe Quantum Dots for Narrow Mid-IR Emission and Detection. J. Am. Chem. Soc. 2011, (41), 16422-16424.

(2) Olmon, R. L.; Slovick, B.; Johnson, T. W.; Shelton, D.; Oh, S.-H.; Boreman, G. D.; Raschke, M. B. Optical Dielectric Function of Gold. Phys. Rev. B 2012, (23), 235147.

(3) Driscoll, W. G.; Vaughan, W.; Optical Society of America. P. 7. In Handbook of optics; McGraw-Hill: New York, 1978.

(4) Weber, M. J. Volume IV: OPTICAL MATERIALS, p. 100. In Handbook of optical materials; CRC Press: Boca Raton, 2003.

(5) Chu, A.; Gréboval, C.; Goubet, N.; Martinez, B.; Livache, C.; Qu, J.; Rastogi, P.; Bresciani, F. A.; Prado, Y.; Suffit, S.; et al. Near Unity Absorption in Nanocrystal Based Short Wave Infrared Photodetectors Using Guided Mode Resonators. ACS Photonics 2019, (10), 2553-2561. 